\title{
Hybrid Contourlet-DCT Based Robust Image Watermarking Technique Applied to Medical Data Management
}

\author{
Sudeb Das and Malay Kumar Kundu \\ Machine Intelligence Unit, Indian Statistical Institute, Kolkata 700 108, India \\ to.sudeb@gmail.com, malay@isical.ac.in
}

\begin{abstract}
This paper describes a non-blind, imperceptible and highly robust hybrid Medical Image Watermarking (MIW) technique for a range of medical data management issues. The method simultaneously addresses medical information security, content authentication, safe archiving and controlled access retrieval. We propose the use of Contourlet Transform (CLT) followed by the Discrete Cosine Transform (DCT) to achieve higher robustness and imperceptibility. Experimental results and performance comparisons confirm the effectiveness and efficiency of the proposed scheme.
\end{abstract}

Keywords: Watermarking, Content Authentication, Data integrity, Contourlet Transform.

\section{Introduction}

The rapid and significant growth of the information and communication technologies has changed the medical data management systems immensely. The modern integrated health-care delivery systems (such as Hospital Management Systems (HISs), Picture Archiving and Communication Systems (PACS) etc.) provide easier access, effective manipulation and efficient distribution of medical data. On the other hand these advances have resulted in new risks of inappropriate use of medical information, due the ease with which digital data can be accessed and manipulated. Therefore, we need a system for effective storage, transmission, controlled manipulation and access of medical data keeping its authenticity, integrity and confidentiality intact [1].

Many MIW techniques have been proposed by various researchers. A spatial domain technique was proposed by Zain et al. 2] to improve the security of medical images by involving the ability to detect tamper and subsequently recover the images. A Region of Interest (ROI) lossless MIW technique with enhanced security and high payload embedding in the spatial domain was proposed by Kundu et al. in [3]. Chao et al. 4]. proposed a DCT based data hiding scheme, capable of hiding EPR in the quantized DCT coefficients of an image. Wu et al. [5] proposed two schemes based on Modulo 256 and DCT, for tamper detection and recovery purpose. Recently few Discrete Wavelet Transform (DWT) based 
MIW methods have been proposed [6] 7] 8] by various researchers. Most of the previous researches have concentrated on preserving the resolution of the medical images after watermark embedding, regardless of testing the robustness of the schemes against different attacks. Moreover, most of the conventional watermarking schemes are semi-robust in nature. They can resist either the signal processing attacks destroying the high frequency $(\mathrm{HF})$ components of the image, or the signal processing operations in which the low frequency (LF) components of the image is altered.

To overcome these problems, we propose a hybrid CLT-DCT based MIW technique to hide Electronic Patient Record (EPR), Doctor's Identification Code (DIC) and Indexing Keyword (INDX) in the medical image. The confidentiality of the EPR is improved by embedding the data in the image. DIC works as a source authenticator and INDX serves as a querying keyword for use in medical image databases for effective image retrieval. In addition to that, both the storage and transmission bandwidth requirements as well as the possibility of EPR detachment are reduced. In the proposed method, watermark's DCT coefficients are inserted into the DCT coefficients of the CLT transformed low pass version of the original image. Due to the special transform structure of Laplacian Pyramid (LP) 9], the DCT coefficients of the watermark are likely to be spread out into all subbands when we reconstruct the watermarked image. As a result the proposed technique is expected to be robust to various attacks resulting from both the signal processing operations which affect the HF or the LF components of the image.

The organization of the paper is as follows: in Section 2 we briefly describe the CLT. We outline in Section 3 the proposed watermark embedding and extraction algorithms. Experimental results and comparisons are presented in Section 4 and we draw conclusion in Section 5.

\section{Contourlet Transform (CLT)}

The major drawback of DWT in two dimensions is their limited ability in capturing directional information. In light of this, Do and Vetterli 9 10 developed the CLT based on an efficient two-dimensional multiscale and directional filter bank (DBF). CLT not only possess the main features of DWT, but also offer a high degree of directionality and anisotropy. It allows for different and flexible number of directions at each scale, while achieving nearly critical sampling. In addition, CLT uses iterated filter banks, which makes it computationally efficient $(O(N)$ operations for an N-pixels image).

CLT gives a multiresolution, local and directional expansion of image using Pyramidal Directional Filter Bank (PDFB). The PDFB combines LP which captures the point discontinuities, with a DFB which links these discontinuities into linear structures. LP is a multiscale decomposition of $L^{2}\left(R^{2}\right)$ into series of increasing resolution subspaces which are orthogonal complements of each other as follows: 


$$
L^{2}\left(R^{2}\right)=V_{j_{0}} \oplus\left(\bigoplus_{j=J_{0}}^{-\infty} W_{j}\right)
$$

An $l$-level DFB generates a local directional basis for $l^{2}\left(Z^{2}\right)$ that is composed of the impulse response of the DFBs and their shifts. In CLT, the directional filter is applied to the detail subspace $W_{j}$. This results in a decomposition of $W_{j}$ into $2^{l_{j}}$ subspaces at scale $2^{j}$.

$$
W_{j}=\bigoplus_{k=0}^{2^{l_{j}-1}} W_{j, k}^{l_{j}}
$$

Fig. 1. (a) shows the flowchart of CLT for a $512 \times 512$ image. A DFB is designed to capture the high frequency content like smooth contours and directional edges. This DBF is implemented by using a k-level binary tree decomposition that leads to $2^{k}$ subbands with wedge shaped frequency partition as shown in Fig. 1].(b).

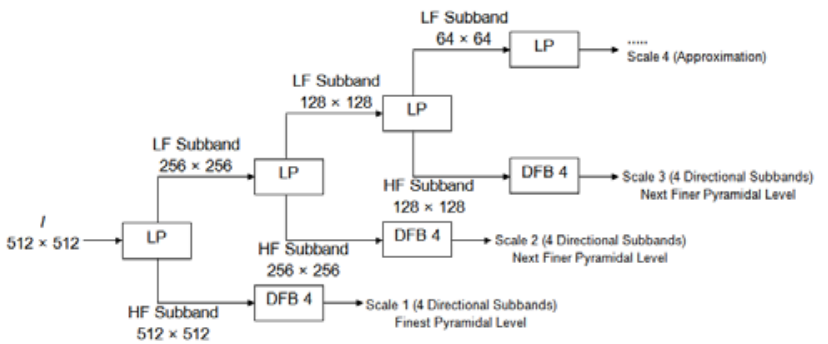

(a)

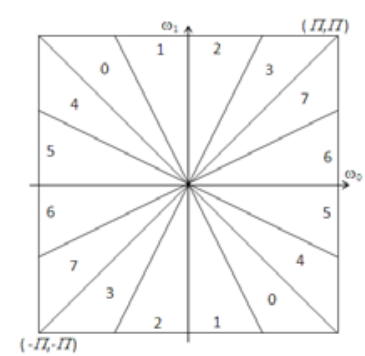

(b)

Fig. 1. (a) Flowchart of CLT for a $512 \times 512$ image. (b) Frequency partitioning $(k=$ $3,2^{k}=8$ ) wedge shaped frequency subbands.

\section{Proposed Scheme}

The watermark embedding and extraction algorithm is described here.

\subsection{Watermark Embedding}

The salient steps of the watermark embedding process are as follows:

1. Apply CLT on the original image $I$.

2. Divide the coefficients of the low pass subband $\left(I_{L P S}\right)$ of the transformed $I$ into $8 \times 8$ non-overlapping blocks.

3. Perform DCT to each block of $I_{L P S}$ to get $D C T_{-} I_{L P S}$.

4. Encrypt the EPR by Advanced Encryption Standard (AES) method using a secret key $K_{S}$ to get $E P R_{E N C R Y}$. 
5. Concatenate $D I C, I N D X, E P R_{E N C R Y}$ and represent the resultant string to its binary representation as $W M_{B I N}$.

6. Apply $\mathrm{BCH}$ error correcting code to $W M_{B I N}$ to get $W M_{B C H}$.

7. Reshape $W M_{B C H}$ as a 1 D-binary string $\left(W M_{B S}\right)$ and scatter its bits using the function given below:

$$
f(x)=p x \bmod n+1
$$

where,

$n=$ size of the subband $I_{L P S}$;

$p=$ secret prime number $\epsilon[1, n]$;

$x=$ bit position in $W M_{B S}$ and $x \in\left[1\right.$, length $\left.\left(W M_{B S}\right)\right]$;

8. Reshape the resultant string in a matrix of equal size as $I_{L P S}$ to get the final watermark payload $W M_{F I N}$.

9. Divide $W M_{F I N}$ into $8 \times 8$ non-overlapping blocks, and perform DCT to each block to get $D C T_{W M}$.

10. Let, a coefficient of the matrix $D C T_{-} I_{L P S}$, is represented by $Y(x, y)$, and $W(x, y)$ represents a coefficient of the matrix $D C T_{W M}$. Then, perform additive watermarking operation:

$$
Y^{\prime}(x, y)=Y(x, y)+\alpha W(x, y)
$$

where,

$Y^{\prime}(x, y)=$ coefficients of the reconstructed matrix $D C T^{\prime} I_{L P S}$;

$\alpha=$ watermarking strength factor.

11. Perform Inverse DCT on $D C T^{\prime} I_{L P S}$, to get the modified $I_{L P S}^{\prime}$.

12. To get the watermarked image $I^{*}$, apply Inverse CLT on the $I_{L P S}^{\prime}$, along with other non-modified directional subbands.

\subsection{Watermark Extraction}

The proposed watermarking scheme is non-blind, which means we need the original image during the extraction process. The steps of the extraction process are as follows:

1. Apply CLT on $I$ and $I^{*}$ (possibly attacked) with the same decomposition configuration of the embedding process.

2. Divide the coefficients of the low pass subbands ( $I_{L P S}$ and $I_{L P S}^{*}$ respectively) of the transformed $I$ and $I^{*}$ into $8 \times 8$ non-overlapping blocks.

3. Get $D C T_{-} I_{L P S}$ and $D C T_{-} I_{L P S}^{*}$ by performing DCT to each block of $I_{L P S}$ and $I_{L P S}^{*}$ respectively.

4. Let, a coefficient of the matrix $D C T \_I_{L P S}$, is represented by $Y(x, y)$, and $Y^{*}(x, y)$ represents a coefficient of the matrix $D C T_{-} I_{L P S}^{*}$. Then, to get one coefficient $W^{\prime}(x, y)$ of the reconstructed DCT watermark $D C T_{W M^{\prime}}$ perform the following operation:

$$
W^{\prime}(x, y)=\left(Y^{*}(x, y)-Y(x, y)\right) / \alpha
$$


5. Perform the Inverse DCT on $D C T_{W M^{\prime}}$, to get the reconstructed watermark $W M^{\prime}$.

6. Reshape $W M^{\prime}$ as a binary string and rearrange the bits to their original positions using the equation 3 to get $W M_{B C H}^{\prime}$.

7. Get $W M_{B I N}^{\prime}$ by applying $\mathrm{BCH}$ decoding procedure on $W M_{B C H}^{\prime}$.

8. Transform $W M_{B I N}^{\prime}$ to its original format and separate $D I C, I N D X$, and $E P R_{E N C R Y}$.

9. Decrypt $E P R_{E N C R Y}$ using the same secret key $K_{S}$ to get the original $E P R$.

\section{Experimental Results and Comparisons}

4 grayscale (8 bpp) medical images of different modalities (CT, MRI, USG), of size $512 \times 512$ were used in the experiments. The $D I C$ and $I N D X$ consist of 20 $(20 \times 7=140 \mathrm{bits})$ and $14(14 \times 7=98 \mathrm{bits})$ ASCII characters respectively. The size of the encrypted EPR was 1152 bits. After applying $\operatorname{BCH}(255,139,15)$ the size of the watermark was 2550 bits. After three level CLT $[2,4,8]$ of the image with ' $9-7$ ' pyramid filter and 'pkva' directional filter, the size of the low pass subband was $64 \times 64$. We used $p=23$ in the equation 3 . The value of $\alpha$ was set to 30 after lots of experiments.

We used Peak Signal-to-Noise Ration (PSNR) to measure the distortion produced after watermark embedding. Mean Structural SIMilarity index (MSSIM) was used to measure the similarity between the original image and the watermarked image. To prove the authenticity of the extracted watermark we used Normalized Correlation (NC) coefficient and Bit Error Rate (BER).

Table 1, shows the PSNR, MSSIM, NC and BER values in attack free case. The result shows that the watermark is retrieved successfully without any errors. Fig. 2, shows the effect of watermark embedding in the medical images. The results show that there is no visual difference between the original and the watermarked images.

The results (for abdomenCT image) in the Table 2, show that the propose method is robust against various image processing attacks. It also shows the visual and structural difference between the watermarked and the attacked image in terms of PSNR and MSSIM.

Comparisons with [7] and [8] reveals that, these methods are more imperceptible (in terms of PSNR), but less robust than our scheme. Specifically, these methods can only survive JPEG compression attack, even that also to a limited

Table 1. Results of Attack Free Case

\begin{tabular}{|c|c|c|c|c|}
\hline Image & PSNR & MSSIM & NC & BER \\
\hline abdomeCT & 41.5895 & 0.8745 & 1.0000 & 0 \\
\hline cthead & 41.5713 & 0.9567 & 1.0000 & 0 \\
\hline mriBrain & 41.5157 & 0.9747 & 1.0000 & 0 \\
\hline usg & 41.4776 & 0.9383 & 1.0000 & 0 \\
\hline
\end{tabular}



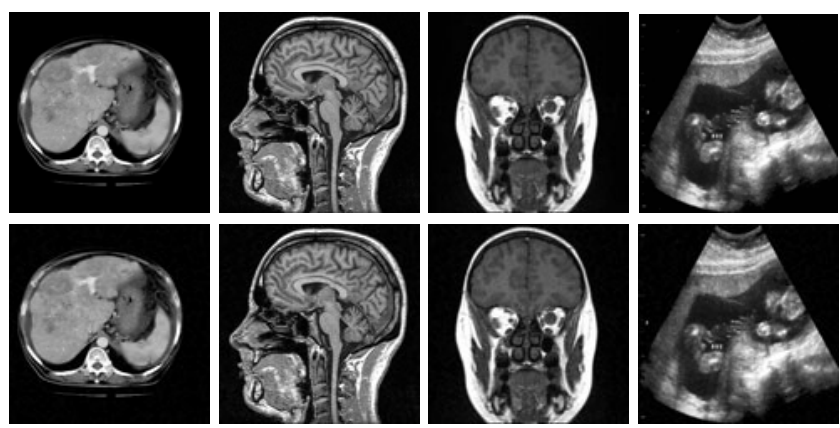

Fig. 2. Original images (above) and their corresponding watermarked images (below)

Table 2. Robustness Against Various Attacks

\begin{tabular}{|c|c|c|c|c|c|}
\hline Attacks & Parameters & NC & BER & PSNR & MSSIM \\
\hline Mean Filtering & Window size $=3 \times 3$ & 1.0000 & 0.0000 & 31.8707 & 0.9434 \\
\hline Median Filtering & Window size $=3 \times 3$ & 1.0000 & 0.0000 & 34.7341 & 0.9555 \\
\hline Salt \& Pepper Noise & Noise density $=0.005$ & 1.0000 & 0.0000 & 26.9014 & 0.8790 \\
\hline Cropping & $315 \times 27$ pixels & 1.0000 & 0.0000 & 20.6878 & 0.9588 \\
\hline Resize & $256 \times 256$ pixels & 1.0000 & 0.0000 & 33.2382 & 0.9525 \\
\hline Bit-Plane Removal & LSB-1 & 1.0000 & 0.0000 & 52.1812 & 0.9936 \\
\hline Sharpening & 65 & 1.0000 & 0.0000 & 29.1512 & 0.9125 \\
\hline JPEG & Quality Factor $=25$ & 1.0000 & 0.0000 & 34.5269 & 0.8819 \\
\hline
\end{tabular}

quality factor $(<50)$. Whereas, our scheme can survive JPEG compression attack up to 25 quality factor. In [7] only the ROI of the image can be embedded, but no EPR or DIC or INDX. Comparison with [6] shows that, our method is more imperceptible (in terms of PSNR) and also more robust. Also the use of EPR encryption, scattering of bits in the embedding process makes our method much more secure than the methods mentioned above.

\section{Conclusions}

We have presented a non-blind, highly robust watermarking scheme applied to medical images with good imperceptibility and enhanced security. Our scheme can be used for different medical image modalities. The experimental results indicate that the proposed scheme is feasible and given its relative simplicity, it can be applied to the medical images at the time of acquisition to serve in many medical applications concerned with authenticity, integrity and confidentiality.

In future research, we will try to enhance our algorithm by making it blind, as well as, we will try to embed the watermark such that, it cause less visual degradation and better extraction accuracy. 


\section{References}

1. Coatrieux, G., Maitre, H., Sankur, B., Rolland, Y., Collorec, R.: Relevance of watermarking in medical imaging, pp. 250-255 (2000)

2. Zain, J.M., Fauzi, A.R.M.: Medical image watermarking with tamper detection and recovery. In: Proc. 28th IEEE EMBS Annual International Conference, pp. 3270-3273 (2006)

3. Kundu, M.K., Das, S.: Lossless roi medical image watermarking technique with enhanced security and high payload embedding. In: Proc. IEEE International Conference on Patter Recognition (ICPR), pp. 1457-1460 (2010)

4. Chao, H., Hsu, C., Miaou, S.: A data-hiding technique with authentication, integration, and confidentiality for electronic patient records. IEEE Trans Inf. Technol. Biomed. 1, 46-53 (2002)

5. Wu, J.H.K., Chang, R.F., Chen, C.J., Wang, C.L., Kuo, T.H., Moon, W.K., Chen, D.R.: Tamper detection and recovery for medical images using near-lossless information hiding technique. Journal of Digital Imaging 21, 59-76 (2008)

6. Manasrah, T., Al-Haj, A.: Management of medical images using wavelets-based multi-watermarking algorithm. In: Proc. Int. Conf. Innovations in Information Technology, pp. 697-701 (2008)

7. Lee, H.K., Kim, H.J., Kwon, K.R., Lee, J.K.: Roi medical image watermarking using dwt and bit-plane. In: Proc. Asia-Pacific Conf. Communica, pp. 512-515 (2005)

8. Giakoumaki, A., Pavlopoulos, S., Koutsouris, D.: Multiple image watermarking applied to health information management. IEEE Trans. on Information Technology in Biomedicine 10, 722-732 (2006)

9. Do, M.N., Vetterli, M.: Contourlets. In: Welland, G.V. (ed.) Beyond Wavelets, Academic Press, New York (2003)

10. Do, M.N., Vetterli, M.: The contourlet transform: An efficient directional multiresolution image representation. IEEE Transactions on Image Processing 14, 2091-2106 (2005) 